\title{
X-ray source populations in star-forming galaxies
}

\author{
Andreas Zezas ${ }^{1}$ \\ ${ }^{1}$ Harvard-Smithsonian Center for Astrophysics, Cambridge, MA 02138, USA \\ email: azezas@cfa.harvard.edu
}

\begin{abstract}
We present a study of the variable X-ray source populations in the Antennae galaxies and a small sample of nearby star-forming galaxies. We find that source variability does not affect the shape of their X-ray luminosity functions. Several of the sources detected in the Antennae exhibit a wide range of spectral variability patterns. Finally, from our study of a small sample of nearby star-forming galaxies, we find a weak indication for variations of the shape of their luminosity functions.
\end{abstract}

Keywords. galaxies: starburst, X-rays: galaxies, X-rays: binaries.

\section{Introduction}

Star-forming galaxies provide a unique environment to study the High Mass X-ray binary populations (HMXBs). Recent studies which have focused on the X-ray luminosity functions (XLFs) of these X-ray source populations have suggested that there is a universal XLF for HMXBs which has a cumulative slope of $\alpha \sim 0.5$. $\dagger$ However, it is still unclear if and how these XLFs depend on the age of the stellar populations and the properties of their host galaxy. For example, Belczynski et al. (2004), based on theoretical modeling of HMXB populations, find that there is a non-monotonic dependence of the XLF slope on the age of the stellar populations.

An important component of the X-ray source populations found in star-forming galaxies are the Ultraluminous X-ray sources (ULXs), which are defined (rather arbitrarily) as sources with luminosities in excess of $10^{39} \mathrm{erg} \mathrm{s}^{-1}$. Their nature, and most importantly their relation with the lower-luminosity X-ray source population, is unclear with possibilities including, Intermediate Mass black-holes (IMBHs), mechanical or relativistic beaming, super-Eddington accretion disks, or simply the upper tail of the regular X-ray binary populations (e.g. Zezas \& Fabbiano, 2002; Miller \& Colbert, 2004).

In order to investigate the nature of X-ray sources associated with young stellar populations, and more specifically address their connection with the stellar populations we embarked on a detailed study of nearby star-forming galaxies. Next we present results from our Chandra monitoring observations of the Antennae galaxies and an age sequence of nearby star-forming galaxies.

$\dagger$ Throughout this contribution the slopes of the XLFs will refer to their cumulative form: $\mathrm{N}(>\mathrm{L})=\mathrm{K} \times \mathrm{L}^{-\alpha}$, where $\mathrm{N}(>\mathrm{L})$ is the number of sources which have higher luminosity than $\mathrm{L} ; \mathrm{K}$ is the normalization of the XLF and $\alpha$ is the slope of the power-law function. However, all fits are performed on the differential form of the XLF. 


\section{The Antennae}

\subsection{The monitoring program}

The Antennae ( $\mathrm{D}=19 \mathrm{Mpc})$, the prototypical merging system of galaxies, hosts one of the largest X-ray source populations observed in a star-forming galaxy, including a large number of ULXs (Zezas \& Fabbiano 2002). In order to study the variability properties and probe the faint end of this population we obtained 8 monitoring observations of the Antennae over a span of two years (Fabbiano et al., 2003).

We detect between 40 and 70 sources in the shortest and the longest exposures respectively, down to a typical luminosity of $\sim 7 \times 10^{37}$. In the coadded exposure (totaling $411 \mathrm{ksec}$ ) we detect 120 sources down to a luminosity of $2-5 \times 10^{37} \mathrm{erg} \mathrm{s}^{-1}$, for low and high background regions respectively.

These monitoring observations allow us to investigate the spectral variability of the rich X-ray source population detected in the Antennae. We find that 43 sources are variable either in short or long timescales, and 21 sources show indications for spectral variability based on their hardness ratios. We find a wide range of spectral variability patterns: sources not showing any correlation between luminosity and spectral hardness; sources becoming softer while their intensity increases and sources showing the opposite behavior (i.e. exhibit hard spectra with increased intensity). We interpret the latter case as analog of the very high state observed in Galactic black-hole binaries and microquasars, where changes in the accretion rate affect the temperature of the accretion disk and its relative strength compared to the power-law component (e.g. Miller et al., 2002). In the relatively narrow energy band of the Chandra spectra $(0.3-8.0 \mathrm{keV})$ these transitions can be interpreted as changes in the power-law slope.

\subsection{Multiwavelength counterparts}

Correlation of the X-ray source catalog with star-cluster catalogs from the HST-WFPC2 observations of the Antennae (Whitmore \& Schweizer 1995; Whitmore et al., 1999), shows that the majority of the X-ray sources is associated with regions of recent star-formation. We note that in this comparison we took special care to match the relative astrometry of the HST and Chandra data using ground based wide field optical images. We find optical associations for 66 out of the 104 X-ray sources which fall within the field of view of the HST observations. Of those 64 are associated with young star clusters (younger than $\sim 25 \mathrm{Myr}$ ) or are in regions of on-going star-formation, manifested by the detection of $\mathrm{H} \alpha$ emission. Only $2 \mathrm{X}$-ray sources are associated with older stellar populations, although 7 sources without optical counterparts are found in the bulge regions of the interacting galaxies. This indicates that the majority of the X-ray sources is most likely HMXBs. Even with the improved relative astrometric calibration we find that a significant number of sources (and 6 out of the 12 ULXs) is not associated with star-clusters but instead they lie in their vicinity. This suggests that these sources were either expelled from their parent clusters (Zezas \& Fabbiano 2002) or that their parent clusters have dissolved in the meantime (Soria 2005).

\section{XLF variability}

These monitoring observations also provide a unique opportunity to investigate the effect of individual source variability on their XLF. In Fig. 1 we show the cumulative XLFs of the seven observations together with their associated errors (including Poisson uncertainties on the number of sources and the luminosity of each source, calculated as described in Zezas \& Fabbiano 2002). All seven XLFs are well fitted by power-law models 

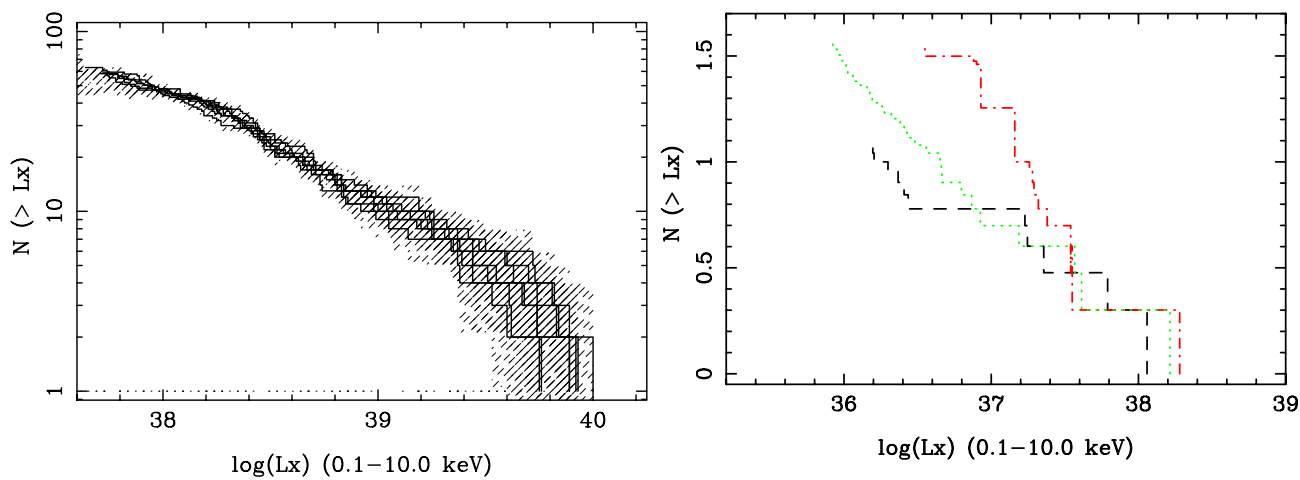

Figure 1. Left: Comparison of the cumulative XLFs from the 7 monitoring observations of the Antennae. The hatched area indicates the uncertainties on each luminosity function (see text). Right: Comparison between the X-ray luminosity functions of NGC 1569 (black, dashed line), the coadded observation of NGC 4214 (green, dotted line) and NGC 5253 (red dash-dot line). All XLFs are corrected for incompleteness.

with consistent slopes of $\alpha \sim 0.5$. A comparison using a Kolmogorov-Smirnov test also shows that they are consistent with a common parent population. This result indicates that X-ray source variability does not affect the shape of the XLFs, which allows us to use single observations of other galaxies in order to obtain a representative picture of their X-ray binary populations.

Since the individual XLFs are consistent with each-other, we fit the XLFs of the coadded observation. We find that to first order it is also fitted with a flat power-law $(\alpha \sim 0.5)$ over three decades in luminosity. There is also indication for a bump at $\sim 3 \times$ $10^{38} \mathrm{erg} \mathrm{s}^{-1}$ and possibly a cutoff at high luminosities, but a preliminary statistical analysis shows that a broken power-law does not provide a better fit than a single powerlaw. The fact that there is no significant difference between the XLF shape of the ULXs $\left(\mathrm{L}_{\mathrm{X}}>10^{39} \mathrm{erg} \mathrm{s}^{-1}\right)$ and the less luminous sources indicates that the ULXs are not associated with a strongly beamed fraction of the "normal" X-ray binary population.

\section{A starburst age sequence}

Although the Antennae offers a large population of X-ray sources, its complicated starformation history results in X-ray binary populations from various stellar generations. This makes it difficult to address the connection between the observed X-ray source populations and their parent stellar populations. For this reason we embarked on a program to study a set of star-forming galaxies with well characterized star-formation histories dominated by a single burst of star-formation. A subset of this sample includes (from younger to older systems) NGC 1569, NGC 4241 and NGC 5253.

In the case of NGC 4241 we have exposures in three different epochs allowing us to look for the effect of source variability on the shape of the XLF. As with the Antennae we did not find any difference between the three XLFs.

In the right panel of Figure 1 we present the XLFs of these three galaxies, corrected for incompleteness. We find that the three XLFs are slightly different, although because of the small number statistics they are fitted with consistent power-law slopes $(\alpha \sim 0.5-$ 0.6). We also see a weak trend for NGC 5253, which has the oldest XRB population, to have the steepest XLF; forthcoming observations will allow us to extend the XLF to lower luminosities in order to test the significance of this trend. 


\section{Discussion and Conclusions}

From our monitoring observations of the Antennae we find that:

- A large number of sources shows intensity and/or spectral variability. In some cases this spectral variability is consistent with spectral changes due to changes in the accretion rate, in the same way as is seen in Galactic sources.

- The majority of the sources is associated with recent star-formation indicating that they are HMXBs. However, a significant number of sources (including 6 ULXs) is not associated with any optically identified star-cluster, indicating either that they were ejected or that their parent star-clusters have dissolved.

- X-ray source variability does not affect the shape of the XLF, allowing us to perform population studies based on individual observations of galaxies.

- There is a weak indication for different XLFs associated with star-forming galaxies with stellar populations of different ages.

\section{Acknowledgements}

I would like to thank my collaborators in the different projects presented in this contribution: G. Fabbiano, F. Schweizer, A. Baldi, V. Kalogera, J. Gallagher, P. Kaaret, A. Prestwich. This work has been supported by NASA LTSA grant NAG5-13056 and Chandra grant G04-5098X.

\section{References}

Belczynski, K., Kalogera, V., Zezas, A., \& Fabbiano, G. 2004, ApJ Lett., 601, L147

Fabbiano, G., Zezas, A., King, A. R., Ponman, T. J., Rots, A., \& Schweizer, F. 2003b, ApJ Lett., 584, L5

Grimm, H.-J., Gilfanov, M., \& Sunyaev, R. 2003, MNRAS, 339, 793

Kilgard, R. E., Kaaret, P., Krauss, M. I., Prestwich, A. H., Raley, M. T., \& Zezas, A. 2002, ApJ, 573,138

Miller, J. M., Wijnands, R., Rodriguez-Pascual, P. M., Ferrando, P., Gaensler, B. M., Goldwurm, A., Lewin, W. H. G., \& Pooley, D. 2002, ApJ, 566, 358

Miller, M. C. \& Colbert, E. J. M. 2004, International Journal of Modern Physics D, 13, 1

Whitmore, B. C., Zhang, Q., Leitherer, C., Fall, S. M., Schweizer, F., \& Miller, B. W. 1999, AJ, 118,1551

Whitmore, B. C. \& Schweizer, F. 1995, AJ, 109, 960

Soria, R. 2005, in Proceedings of XXII Texas Symposium on Relativistic Astrophysics, Stanford University, 2004, (astro-ph/0503340)

Zezas, A. \& Fabbiano 2002, ApJ, 577, 726

\section{Discussion}

MCBreEn: In the Antennae there are heavily obscured regions of star-formation as revealed in the mid-infrared with ISO. What X-ray sources are associated with these regions?

ZEZAS: There are a couple of sources associated with the heavily obscured overlap region between the two galaxies; these sources also show heavily absorbed X-ray spectra. From the same region we see hard diffuse emission which most probably is unresolved emission from X-ray binaries in this obscured, actively star-forming region. On the other hand we do not find many X-ray sources in the inner part of the spiral arm where there is also some dust obscuration. Also most of the ULXs are found in the outer edge of the spiral arm, rather than the highly obscured regions. 